ARTÍCULO

\title{
Evaluación de Aprovechamiento y Propuesta de Clasificación de Madera de Desecho de Nogal Común (Juglans regia)
}

\author{
Loewe, Verónica1*; Bozo, Alejandro² y Jaime, Nicolás ${ }^{3}$ \\ ${ }^{1}$ Investigadora, Instituto Forestal, Sede Metropolitana, Santiago. vloewe@infor.cl \\ ${ }^{2}$ Departamento Ingeniería de la Madera. Universidad de Chile. abozo@uchile.cl \\ ${ }^{3}$ nicolasjaime.moreno@gmail.com \\ Autor para correspondencia vloewe@infor.cl
}

DOI: https://doi.org/10.52904/0718-4646.2021.471

Recibido: 10.04.21; Aceptado: 20.04.21

\section{RESUMEN}

El nogal común (Juglans regia L.) posee una madera tradicionalmente demandada por el mercado europeo, pero a nivel nacional no existe una cultura de uso de esta madera, por lo que las trozas obtenidas en los raleos se pierden como desecho. Para resolver este problema se desarrolló una investigación con el objetivo de evaluar el rendimiento en el aserrío de trozas de raleo de nogal y desarrollar una propuesta de clasificación para las piezas aserradas obtenidas. Se utilizaron 74 trozas, con edades entre 7 y 11 años. Se determinó el volumen de madera que ingresó al aserradero, utilizando la fórmula de cubicación de trozas de Smalian, luego se aserraron las trozas obteniendo piezas con caras lisas y cantos muertos, de las cuales se obtuvieron tablillas de escuadrías variables, a las que se les determinó el volumen, con lo que se pudo calcular el rendimiento del proceso. Además, para analizar los defectos en las trozas y su relación con el rendimiento, estas se dividieron en dos grupos, utilizando una norma brasileña de clasificación de trozas. Junto a lo anterior, se desarrolló una clasificación para estas tablillas utilizando como base la normativa chilena, basada en el criterio visual. Como resultado se obtuvo un rendimiento del $15,14 \%$, con tablillas de volúmenes entre 0,0011 y $0,0051 \mathrm{~m}^{3}$ por tablilla, clasificadas en tres grupos, concluyéndose que es posible obtener madera aserrada a partir de trozas de raleo de plantaciones jóvenes.

Palabras clave: Nogal, Valorización de madera, defectos, madera aserrada, raleo, rendimiento en aserrío.

\begin{abstract}
SUMMARY
Walnut (Juglans regia L.) has a timber traditionally demanded by the European market, but at the national level, there is no culture of use of this timber, so logs obtained from thinning are lost as waste. To solve this problem, an investigation was carried out with the objective of evaluating the saw performance of thinned Walnut logs, and developing a classification proposal for the sawn pieces obtained. The volume of wood that entered the sawmill was determined using the Smalian log cubing formula, then the logs were sawn obtaining pieces with smooth faces and edges with bark, from which small boards were sized. Volume of these small pieces was calculated, and finally the yield of the process was determined; 74 logs were used, with ages between 7 and 11 years. Log defects and their relation to the yield were in addition analyzed; logs were classified into two groups using a Brazilian norm for log classification. It was also developed a classification system for Walnut small boards using Chilean regulations as a basis, based on visual criteria. As a result, a yield of 15.14 $\%$ was obtained, with volumes for small boards ranging between 0.0011 and $0.0051 \mathrm{~m}^{3}$ each, classified into three groups, concluding that it is possible to obtain sawn wood from logs from young plantations thinning's.
\end{abstract}

Key words: Walnut, wood recovery, defects, sawn timber, thinning, sawmill performance.

\section{INTRODUCCIÓN}

El nogal común (Juglans regia L.) es un árbol vigoroso, longevo, de tronco recto, cultivado en Europa, Asia y Norteamérica. Produce un fruto muy valioso (nueces) y presenta la ventaja de ser una especie conocida y estar arraigada en el país. Además, posee una madera muy apreciada para distintas aplicaciones, como carpintería, ebanistería, tornería, artesanía y otras. Esta variada utilización ha permitido el desarrollo de diversos mercados, destacando el italiano y el francés por los altos precios que ha llegado a obtener esta madera, la que incluso ha sido considerada en ciertos períodos como la más cotizada en Europa (Loewe y González, 2001).

En Chile no existe un mercado para la madera de nogal, principalmente por una falta de cultura de uso de esta madera y por el reducido poder adquisitivo de la población media, además de la nula producción 
maderera, ya que aún no hay cosechas finales, mientras que la obtenida en los raleos se utiliza principalmente como combustible, aunque se han explorado otras alternativas para valorizarla (Álamos, 2003).

Para resolver este problema se ha propuesto generar una clasificación para la madera aserrada obtenida de raleos de plantaciones jóvenes, con el fin de determinar posibles usos para esta madera, lo que permitirá dar un uso alternativo y de mayor interés comercial que el de combustible.

\section{MATERIALES Y MÉTODO}

El estudio se realizó el año 2006 (Jaime, 2006), y utilizó 74 trozas con edades entre 7 y 11 años, obtenidas de raleos realizados en tres plantaciones de arboricultura que posee la Compañía Agrícola y Forestal El Álamo en predios de la comuna de Retiro, región de Maule, a las que se cuantificó los diámetros sin corteza y el largo, para determinar el volumen total de madera mediante la fórmula para volumen cúbico de Smalian (ecuación [1]), y mediante la sumatoria de todos los volúmenes se obtuvo el volumen total de madera que ingresó a la etapa de aserrío.

$$
V=\frac{(A+a)}{2} * L
$$

Donde:

V: Volumen de la troza $\left(\mathrm{m}^{3}\right)$.

A: Área de la sección mayor $\left(\mathrm{m}^{2}\right)$.

a: Área de la sección menor $\left(\mathrm{m}^{2}\right)$.

$\mathrm{L}$ : Largo de la troza $(\mathrm{m})$.

Junto a lo anterior, se constató la presencia de distintos defectos en las trozas, los que podían afectar la valorización y la utilización de las mismas. Entre los defectos observados se encuentran la excentricidad, rajadura, fisura estrellada, contrafuertes, heridas, grietas por frío, fibra helicoidal, pudrición, ahusamiento y madera de reacción, determinándose su presencia en las trozas.

Además se cuantificó la existencia de ovalización mediante la medición de diámetros en las caras de la troza; curvatura, que se cuantificó midiendo la distancia existente entre el eje recto que une las dos caras y el eje que presentaba la troza; ahusamiento, calculado como la diferencia entre los diámetros mayores y menores, dividiéndose luego por el largo de la pieza; y la regularidad de anillos, que se determinó mediante el cálculo de la diferencia entre la distancia mayor y la distancia menor de los anillos de crecimiento; si la diferencia era superior a $1 \mathrm{~cm}$ se consideraron irregulares.

Luego, basados en la Norma Brasileña para la Medición y Clasificación de Trozas (Góngora y Serrano, 2006), se clasificaron las trozas, según la ecuación [2], para facilitar el análisis de los efectos que generaban los distintos defectos en las trozas, respecto a la cantidad y calidad de la madera obtenida.

$$
F C=\left\{\left[[(A+E x M) / 2]+\left[(C+C u)^{*} 2\right] / 2\right] / 2\right\} / 10
$$

Donde:

FC: Factor de clasificación

A: Ovalización (cm)

ExM: Excentricidad de la Médula (cm)

C: Conicidad $(\mathrm{cm} / \mathrm{m})$

$\mathrm{Cu}$ : Curvatura $(\mathrm{cm})$ 
La clasificación de las trozas se realizó a partir del factor de clasificación promedio obtenido, dividiéndolas en dos grupos, aquellas con un factor bajo el promedio, y aquellas con un factor mayor al promedio. Los primeros corresponderán a las trozas de mejor calidad, llamadas calidad A, mientras las segundas corresponden a trozas de calidad B.

El aserrío se realizó con sierras huincha de 2 pulgadas de ancho y 1,76 $\mathrm{mm}$ de espesor; para realizar los cortes tangenciales en la troza y obtener las tapas de madera. Las tapas obtenidas fueron dibujadas para determinar las tablillas según una técnica indicada por Servin y Lemaire (2004) utilizada por aserraderos de castaño en Francia, llamada técnica de despachos sobre listas, la cual consiste en delinear en sentido longitudinal para eliminar los cantos muertos o médula, obteniendo lados paralelos, y trozar para eliminar los defectos obteniendo piezas variables en función de las posibilidades del recurso; este proceso se realizó para obtener los mejores niveles de calidad posibles de cada tapa.

Una vez dibujadas, las tapas pasaron por una sierra circular de 5,18 $\mathrm{mm}$ de espesor, para realizar el trozado y dimensionado de las tablillas dibujadas.

Una vez aserradas, las piezas obtenidas fueron medidas para determinar el volumen luego del procesamiento; esta medición se realizó cuantificando los largos, anchos y espesores de las piezas aserradas, y luego multiplicando todas estas variables se obtuvieron los volúmenes $\left(\mathrm{m}^{3}\right)$, de madera de cada pieza, y mediante su sumatoria se determinó el volumen total de madera obtenida.

Para desarrollar la clasificación se utilizó como base la normativa chilena, desarrollando una clasificación por aspecto, que corresponde a una clasificación efectuada considerando la apariencia de la madera como principal requisito de acuerdo al uso a que se destine. El procedimiento utilizado para el desarrollo de la clasificación, como lo señala la norma NCh 993 EOf 72, consideró los siguientes pasos:

- Determinar los defectos que interesa separar o delimitar en la clasificación.

- Fijar el número de grados que se desea establecer; en esta etapa se determinó el número de niveles a desarrollar, que en este caso fueron 3.

- Determinar, para cada grado y cada defecto, el valor admisible del defecto de modo que fuera conveniente al uso a que se destinaría la madera, es decir, se determinó el valor aceptable para cada defecto por nivel.

- Seleccionar los niveles o valores admisibles, para cada defecto, tomando en cuenta la especie, el defecto y el grado considerado, es decir, se determinó el rango en el cual el defecto se considera admisible en cada nivel.

Dentro de los defectos típicos que se presentan en las piezas semi-elaboradas de nogal y que fueron considerados en el estudio constatando su presencia, se encuentran: tensiones de crecimiento, ataque de hongos y veteado negro. Otros defectos, que fueron estimados como lo indica la norma NCh 992 EOf 72, son: nudos firmes o sueltos, nudos agrupados, arista faltante, torcedura, arqueadura, encorvadura y acanaladura. Los datos fueron tabulados en Excel y mediante la ecuación [3] se determinó el rendimiento obtenido por el aserradero.

$\mathrm{R}=\frac{\mathrm{VMA}}{\mathrm{VT}}{ }^{*} 100$

Donde:

R: Rendimiento (\%)

VMA: Volumen de Madera Aserrada $\left(\mathrm{m}^{3}\right)$

VT: Volumen Total de madera $\left(\mathrm{m}^{3}\right)$ 


\section{RESULTADOS}

\section{Características de las Trozas}

En total, el volumen de madera es de $1,03293 \mathrm{~m}^{3}$, con un promedio de $0,01396 \mathrm{~m}^{3}$ por troza (Cuadro 1).

La distribución diamétrica de las trozas sin corteza (Figura 1) muestra que el 51,35\% de las trozas se encuentran bajo 10,9 cm de diámetro, dado que los espesores de corteza variaron entre 0,1 y 0,7 cm en el radio, generando una disminución en los diámetros entre 0,4 y $1,4 \mathrm{~cm}$.

En cuanto al aserrío, se obtuvo un total de 342 tapas de nogal en su etapa primaria, con un promedio de 4,62 tapas por troza. Del total de tapas obtenidas en esta etapa, sólo se utilizaron 310, ya que, por algunos defectos como médula, pudriciones y otros se descartaron las restantes 32 .

Cuadro 1. Resumen de las Características Dasométricas de las Trozas

\begin{tabular}{lcccc} 
& $\begin{array}{c}\text { Diámetro Mayor } \\
(\mathbf{c m})\end{array}$ & $\begin{array}{c}\text { Diámetro Menor } \\
\mathbf{( c m})\end{array}$ & $\begin{array}{c}\text { Largo } \\
\mathbf{( m )}\end{array}$ & $\begin{array}{c}\text { Volumen Troza } \\
\left(\mathbf{m}^{\mathbf{3}}\right)\end{array}$ \\
\hline Mínimo & 7,50 & 5,30 & 0,79 & 0,02946 \\
Máximo & 20,10 & 13,60 & 2,53 & 0,00511 \\
Promedio & 12,12 & 9,16 & 1,72 & 0,01396 \\
Desviación Estándar & 2,84 & 1,61 & 0,38 & 0,00536 \\
\hline
\end{tabular}

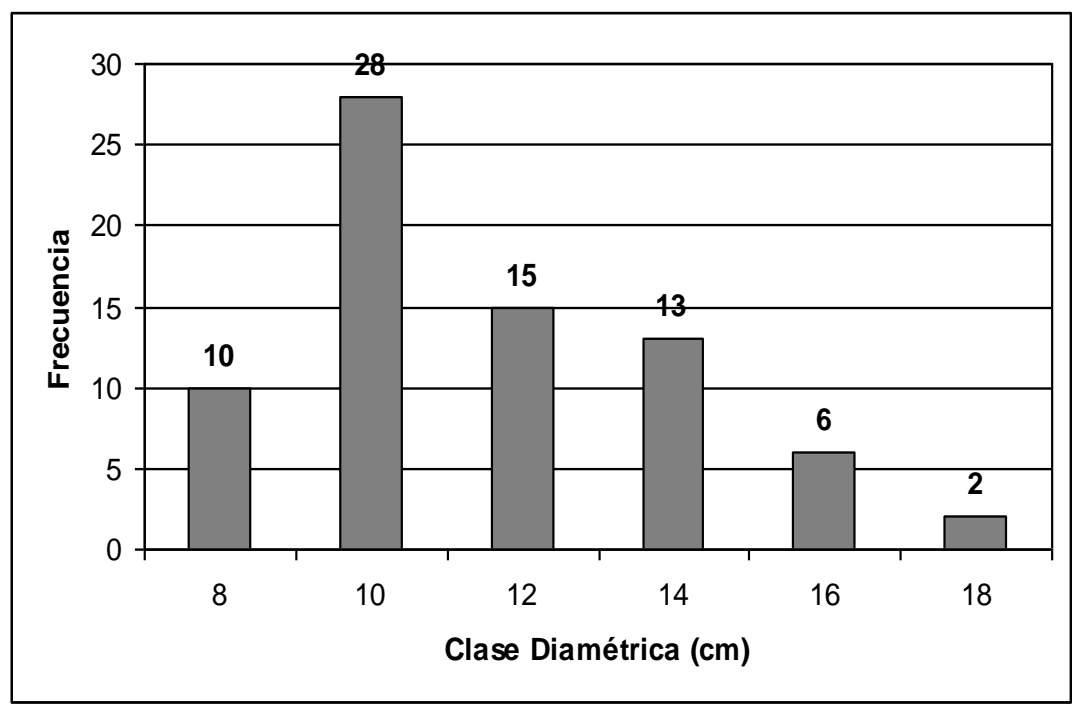

Figura 1. Gráfico de Distribución Diamétrica de Sección Mayor sin Corteza

\section{Rendimiento y Defectos de las Trozas}

Del total de madera ingresado al aserradero, solo $0,15643 \mathrm{~m}^{3}$ se obtuvo como madera aserrada en forma de tablillas; este volumen se dividió en 381 tablillas, que poseen un volumen promedio de $0,00041 \mathrm{~m}^{3}$ por tablilla (Cuadro 2), lo que determina un rendimiento del $15,14 \%$. 
Cuadro 2. Resumen de las Características de las Tablillas

\begin{tabular}{lcccc}
\cline { 2 - 5 } & $\begin{array}{c}\text { Largo } \\
\mathbf{( c m )}\end{array}$ & $\begin{array}{c}\text { Espesor } \\
\mathbf{( c m})\end{array}$ & $\begin{array}{c}\text { Ancho } \\
\mathbf{( c m})\end{array}$ & $\begin{array}{c}\text { Volumen } \\
\left(\mathbf{m}^{\mathbf{3}}\right)\end{array}$ \\
\hline Mínimo & 117,1 & 1,9 & 11,0 & 0,00112 \\
Máximo & 15,1 & 1,0 & 3,0 & 0,00011 \\
Promedio & 47,7 & 1,5 & 5,9 & 0,00041 \\
Desviación Estándar & 20,25 & 0,08 & 1,68 & 0,00018 \\
\hline
\end{tabular}

La pérdida de madera por generación de aserrín correspondió a un 18,23\% del volumen total, donde el aserrín generado por las sierras circulares en el dimensionado correspondió a un 3,1\% del volumen total, mientras el porcentaje generado por las sierras huincha es un $15,1 \%$ del volumen total. La relación entre el diámetro mayor versus el volumen aprovechado (Figura 2) muestra una tendencia ascendente, mientras que la clasificación de las trozas obtuvo como factor de clasificación promedio un 0,374 , lo que permitió dividirlas en dos grupos, calidad $A$, que eran las trozas de mejor calidad, y calidad B. En la calidad A calificaron 37 trozas, mientras en la B también, también 37 trozas, pero los volúmenes y rendimientos fueron distintos (Figura 3).

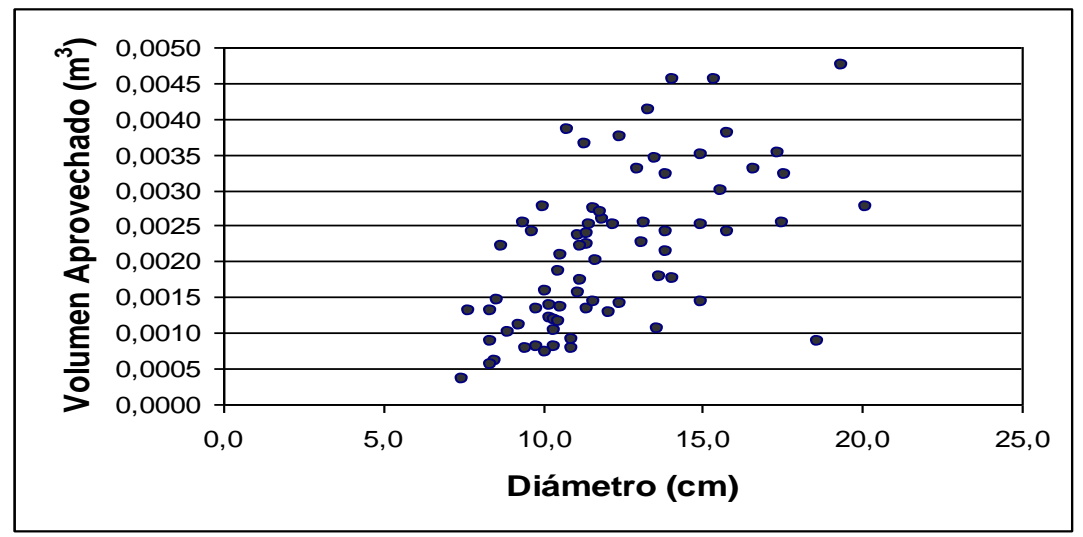

Figura 2. Gráfico de Diámetro vs el Volumen de Madera Aserrada Obtenida

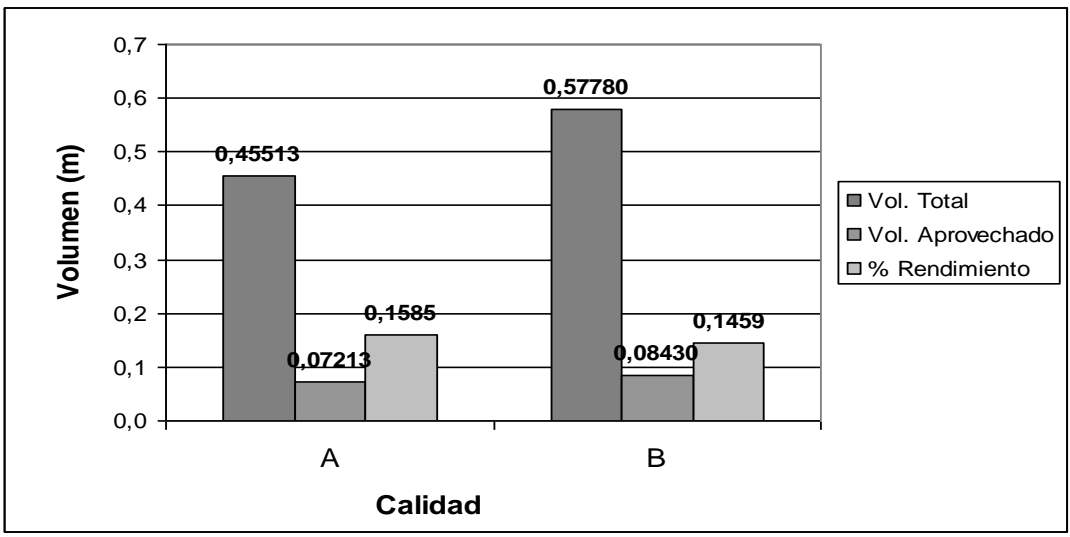

Figura 3. Gráfico de Aprovechamiento y Rendimiento de Trozas Según Calidad 
Respecto a los valores de los defectos utilizados para la clasificación de las trozas (Cuadro 3), demuestran que no se presentó excentricidad en ninguna troza, mientras las mayores diferencias correspondieron a la curvatura, seguida por la excentricidad. Además de estos defectos, existen otros que se presentaron en las trozas de ambas calidades (Figuras 4 y 5 ).

Cuadro 3. Características de las Calidades A y B de Trozas

\begin{tabular}{lc|cc}
\hline \multicolumn{1}{c}{ Defecto } & Unidad & Calidad A & Calidad B \\
\hline Ovalización & $\mathrm{cm}$ & $>2,0$ & $>2,7$ \\
Curvatura & $\mathrm{cm}$ & $>5,1$ & $>22,5$ \\
Ahusamiento & $\mathrm{cm} / \mathrm{m}$ & $>3,8$ & $>5,3$ \\
Excentricidad & $\mathrm{cm}$ & 0 & 0 \\
\hline
\end{tabular}

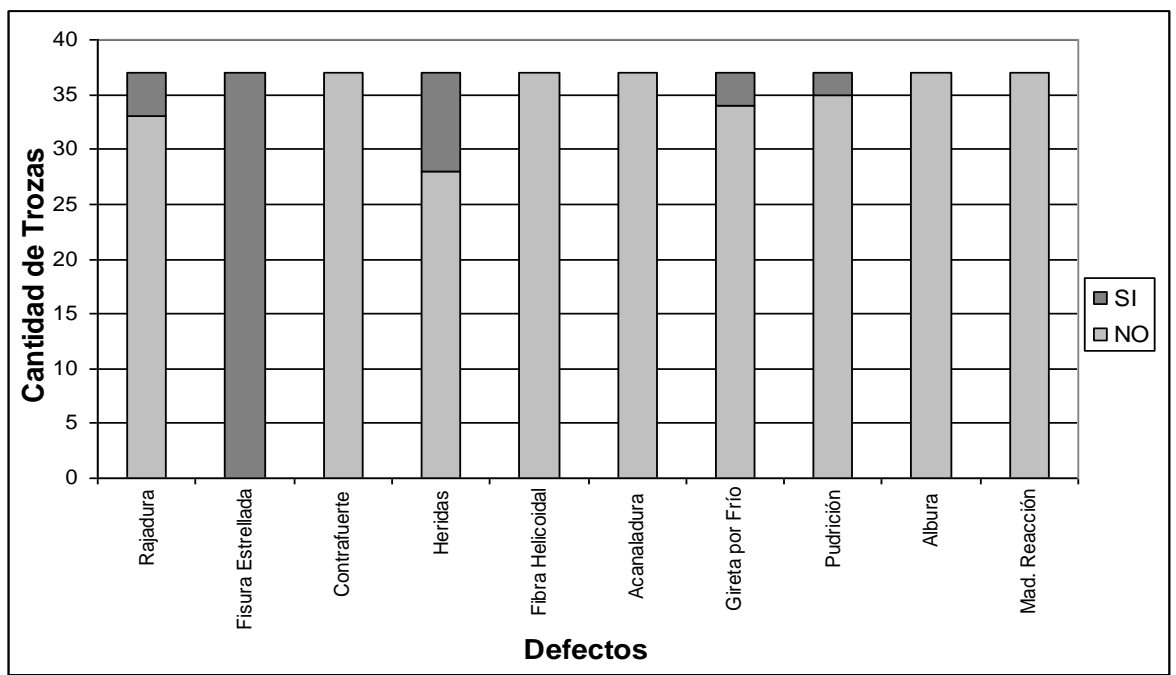

Figura 4. Presencia de Defectos en Trozas de Calidad A

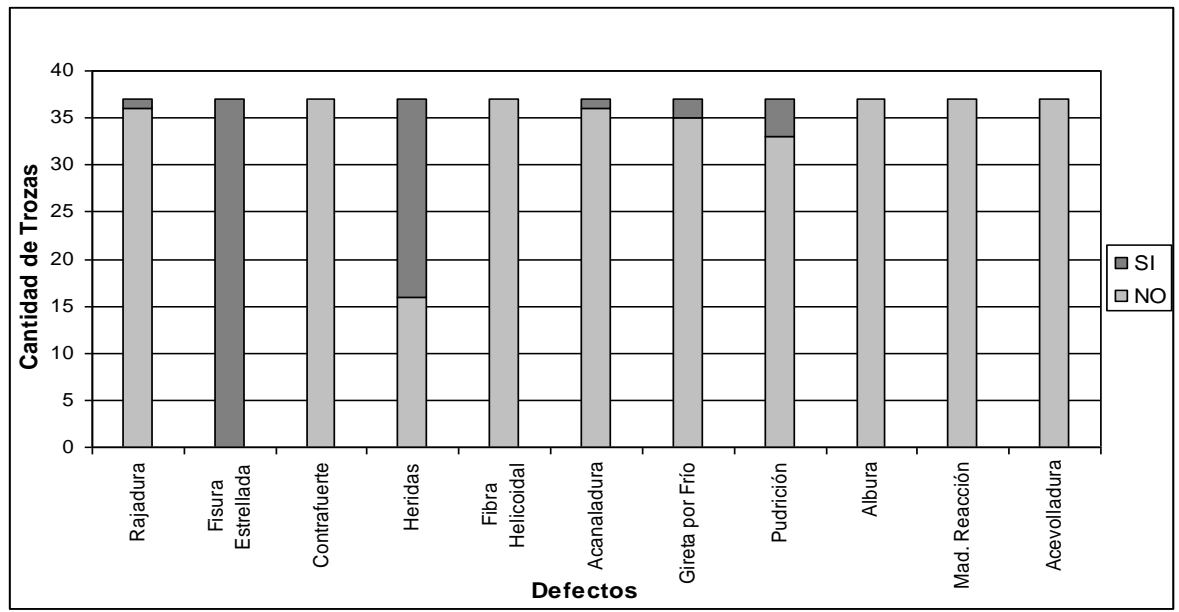

Figura 5. Presencia de Defectos en Trozas de Calidad B 


\section{Clasificación y Características de la Madera Aserrada}

La clasificación (Cuadro 4) presenta los valores aceptados para cada defecto en cada grado.

El grado N1 tiene como posibles usos la aplicación en diversos productos, como elementos decorativos, fabricación de piezas de muebles, y finalmente para producir cajas de embalajes de alto valor estético.

El grado N2 tiene como objetivo usos similares al primer grupo, pero en dimensiones menores, mientras que el grado N3 está pensado para la producción de artesanías y elementos de pequeñas dimensiones, como lápices y otros.

El menor volumen total de tablillas por grupo lo presentó el grado N1, mientras el mayor volumen de madera obtenida correspondió al grado N2 (Cuadro 5).

Cuadro 4. Propuesta de Clasificación Visual para Tablillas

\begin{tabular}{|c|c|c|c|c|}
\hline \multirow{2}{*}{ Defectos } & \multirow{2}{*}{ Unidades } & \multicolumn{3}{|c|}{ Grados } \\
\hline & & N1 & N2 & N3 \\
\hline Largo & $\mathrm{cm}$ & $>30$ & $>20$ & $>10$ \\
\hline Ancho & $\mathrm{mm}$ & $>60$ & $>50$ & $>20$ \\
\hline Espesor & $\mathrm{mm}$ & $>12$ & $>8$ & $>4$ \\
\hline Médula & Presencia & no & no & no \\
\hline Ataque Hongos & Presencia & no & no & no \\
\hline Pudrición & Presencia & no & no & no \\
\hline Nudo c/ pudrición & $\%$ largo & 0 & 0 & 0 \\
\hline Nudo Fijo Caras & $\%$ largo & $<10 \%$ & $<20 \%$ & $<40 \%$ \\
\hline Nudo Fijo Cantos & $\%$ largo & $<5 \%$ & $<15 \%$ & $<25 \%$ \\
\hline Nudos Sueltos Caras & $\%$ largo & $<5 \%$ & $<15 \%$ & $<30 \%$ \\
\hline Nudos Sueltos Cantos & $\%$ largo & $<5 \%$ & $<10 \%$ & $<20 \%$ \\
\hline Veteado Negro & Presencia & no & no & no \\
\hline Rajadura & $\%$ largo & $<5 \%$ & $<15 \%$ & $<25 \%$ \\
\hline Arista Faltante Cara & $\mathrm{mm}$ & $<7$ & $<15$ & $<30$ \\
\hline Arista Faltante Canto & $\mathrm{mm}$ & $<4$ & $<6$ & $<10$ \\
\hline Torcedura & $\mathrm{mm}$ & 0 & $<10$ & $<50$ \\
\hline Acanaladura & $\mathrm{mm}$ & 0 & $<10$ & $<30$ \\
\hline Arqueadura & $\mathrm{mm}$ & 0 & $<10$ & $<40$ \\
\hline Encorvadura & $\mathrm{mm}$ & 0 & $<10$ & $<30$ \\
\hline Regularidad de Anillos & $\mathrm{mm}$ de diferencia & $<5$ & $<10$ & $<25$ \\
\hline
\end{tabular}


Cuadro 5: Resumen de Volúmenes de Piezas Aserradas Clasificadas 'por Grado

\begin{tabular}{lccc}
\cline { 2 - 4 } & Grado N1 & Grado N2 & Grado N3 \\
\hline Vol. Mínimo & 0,00025 & 0,00013 & 0,00011 \\
Vol. Máximo & 0,00106 & 0,00209 & 0,00112 \\
Vol. Promedio & 0,00051 & 0,00047 & 0,00034 \\
Des. Estándar & 0,00021 & 0,00022 & 0,00015 \\
\hline Vol. Total & $\mathbf{0 , 0 1 4 4 1}$ & $\mathbf{0 , 0 8 4 7 8}$ & $\mathbf{0 , 0 5 5 6 9}$ \\
\hline
\end{tabular}

Con relación al tipo de defecto que afectó la clasificación de las piezas (Figuras 6 y 7), se aprecia que en el grado N2 el defecto más recurrente es la dimensión de la pieza con un $59,76 \%$, seguido por los nudos con pudrición y en tercer lugar la irregularidad de los anillos, mientras que el defecto que presentó una menor presencia fue le encorvadura.

En el grado N3, el principal defecto que limitó la clasificación de las piezas fue el tamaño, con un 78,66\% de las piezas, seguido por los nudos con pudrición y la rajadura.

Respecto a las características estéticas de las piezas aserradas, estas no presentaron un proceso de duraminización, además se observaron 4 tonalidades distintas de la madera (Figura 8); estos colores, agrupados según la clasificación de Jay-Allemand et al. (1998), corresponden a colores claros, siendo los colores 1 y 4 considerados grisáceos y correspondieron a un $30,71 \%$ del volumen aserrado, mientras los colores 2 y 3 son catalogados como blancos, y se presentaron en el 69,29\% del volumen aserrado.

Dentro de la calidad N1, el color que dominó fue el blanco, con un $89,3 \%$ del volumen, mientras que en el grado N2 el color que se presentó mayoritariamente fue el grisáceo, con un 72,72\% del volumen.

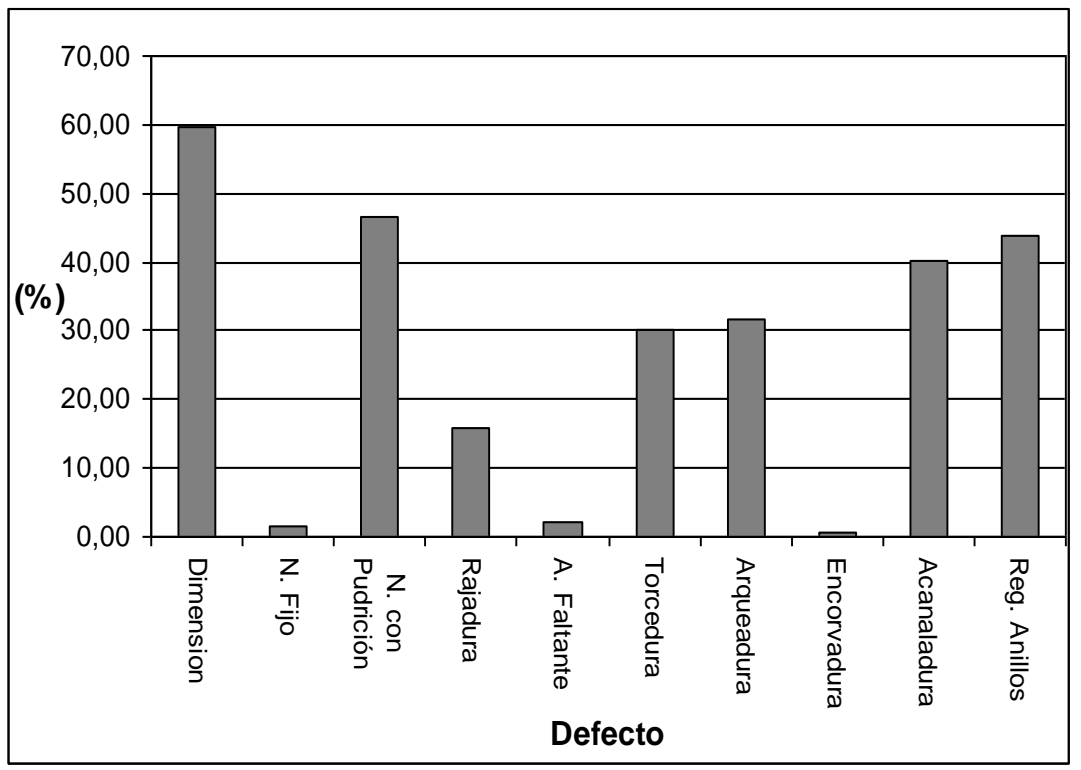

Figura 6. Defectos que Afectaron la Clasificación de las Piezas Aserradas N2 


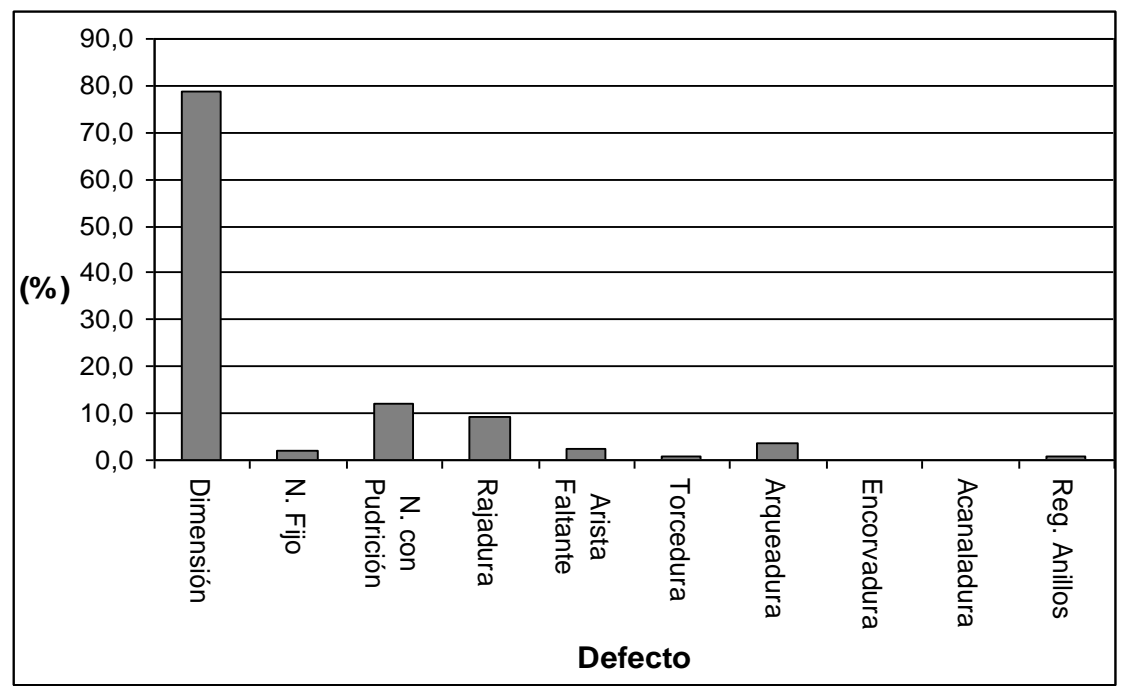

Figura 7. Presencia de Defectos que Afectan la Clasificación de Piezas Aserradas N3

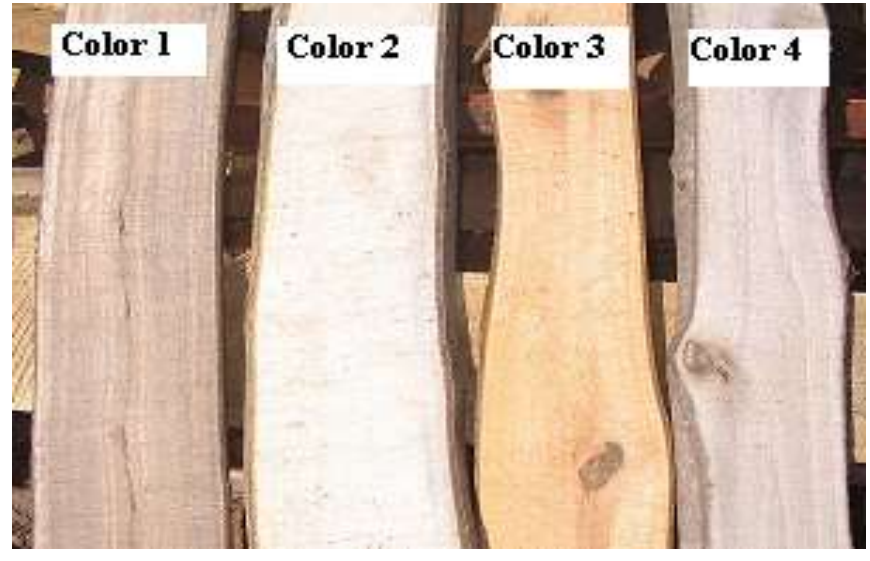

Figura 8. Clasificación de los Colores de la Madera de las Trozas

\section{DISCUSIÓN}

\section{Características de las Trozas}

Las trozas estudiadas presentaron diámetros muy reducidos dada su edad, lo que también se ha reportado en otros estudios como el de Zanuttini et al. (2006), donde las trozas de nogal de 7 años presentaban diámetros entre 8 y $10 \mathrm{~cm}$, pero a diferencia de ese estudio, las trozas se aserraron pese a que los diámetros de las secciones superiores presentaban un mínimo inferior a los valores que comúnmente se utilizan en los aserraderos de diámetros reducidos $(18 \mathrm{~cm})$, lo que limita su potencial maderero, por lo que es necesario analizar otros usos para mejorar el aprovechamiento de estas trozas, u otros métodos de transformación, como el foliado en pequeñas dimensiones.

\section{Rendimiento y Defectos de las Trozas}

Respecto al rendimiento obtenido por el proceso (15,14 \%) es un valor bajo según Fassola et al. (1999), quienes así lo indican para rendimientos bajo 34\%; pero sería lo esperable, ya que Serrano (1996) citado por Góngora y Serrano (2006), indican que el rendimiento en productos aserrados de madera de diámetros pequeños, que oscilan entre 15 y $40 \mathrm{~cm}$, es relativamente bajo (25 - 50\%). Además, otro factor que incide sobre el rendimiento es la técnica de dibujado de las tablillas, ya que el porcentaje de 
desechos producidos es mayor con la técnica utilizada de despachos sobre listas, donde los rendimientos no superan el $50 \%$ de la madera cosechada (Sevrin y Lemaire, 2004).

Además, las dimensiones de las piezas aserradas obtenidas, al ser más pequeñas, requieren de una mayor cantidad de cortes en las tapas, aumentando las pérdidas por formación de aserrín. Es así como Góngora y Serrano (2006) obtuvieron rendimientos de hasta 19\% en la obtención de tablillas, pese a lo cual el total de perdida por generación de aserrín (18,23\% del volumen total) se encuentra dentro de los rangos esperados para un proceso de aserrío, por lo que, si bien las dimensiones de las tablillas son un factor determinante en el rendimiento, este no es tan importante como la técnica de dibujado de tablillas.

Otro elemento que afecta el rendimiento es el tipo de sierra utilizado según los diámetros, en este caso las sierras utilizadas no corresponden a las indicadas para diámetros pequeños, además, las sierras huinchas en algunos casos se curvaban durante el aserrado, torciendo el corte (Figura 9) y generando escuadrías irregulares, esto porque el aserradero utilizaba principalmente Pinus radiata, cuya madera poseen una menor dureza. Aún así, y para aumentar en algún grado el rendimiento, es recomendable la realización de una evaluación de rendimiento con la utilización de un aserradero que trabaje con diámetros reducidos.

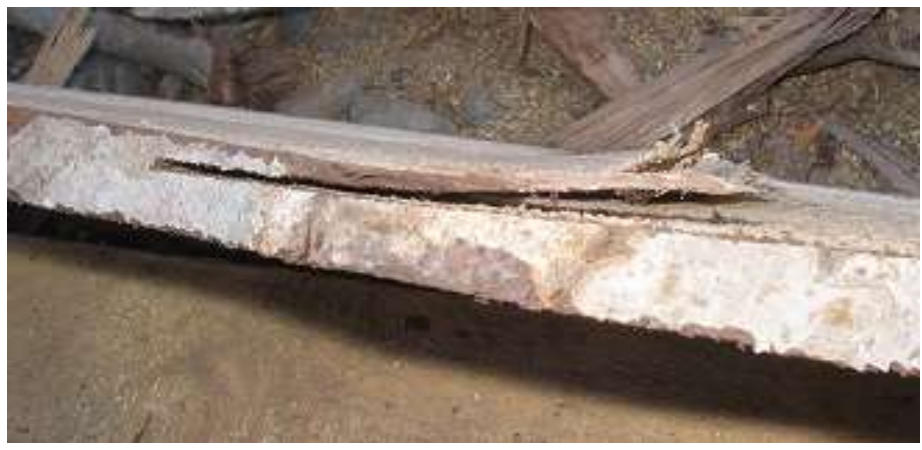

Figura 9. Curvado en el Corte de Tapas

Álvarez et al. (2005) indican que a medida que aumenta el diámetro de las trozas se incrementan los rendimientos (Figura 2), lo que se aprecia levemente ya que no se distingue una verdadera línea sino una dispersión de puntos con cierto ordenamiento ascendente. Además, se observa que al aumentar el volumen la variabilidad del volumen aprovechable aumenta, lo que indica que los defectos tienen gran incidencia en el rendimiento de las trozas, sobre todo si el defecto imposibilita la utilización de un área de la pieza como madera aserrada, como es el caso de la pudrición, heridas y grietas.

Lo anterior se aprecia en que las trozas con los factores de clasificación más bajos (calidad A) obtuvieron un mayor rendimiento (Figura 3), siendo este superior en 1,26 puntos porcentuales al obtenido por las trozas de peor calidad (calidad B), existiendo además un rendimiento mayor al total obtenido en 0,71 puntos porcentuales; para explicar esto se debe analizar las características de cada grupo (Cuadro 3), apreciándose que la mayor diferencia se presenta en la curvatura, seguida por el ahusamiento, mientras que la excentricidad de la médula es el único factor que no afectó la clasificación, ya que no se presentó en las trozas.

La diferencia en la curvatura es clave a la hora de encontrar una causa para la diferencia de rendimientos, ya que Álvarez et al. (2005) reconocen la existencia de una regla general que indica que un incremento de 0,1 en la proporción torcedura-diámetro conduce al decrecimiento del rendimiento volumétrico en un $5 \%$ en la troza; entonces, al existir curvaturas mayores es más fácil alcanzar y sobrepasar este valor, por lo que es deseable obtener trozas sin curvatura, siendo aceptable una curvatura de menos de $5 \mathrm{~cm}$ ya que permite obtener un rendimiento mayor al esperado por el proceso 
(15,14\%). Lo mismo ocurre para la ovalización y el ahusamiento, pero con valores de $2,1 \mathrm{~cm}$ y $3,8 \mathrm{~cm} / \mathrm{m}$, respectivamente.

Otros defectos en las trozas (Figuras 4 y 5 ) también tienen efecto en el rendimiento. Los defectos más frecuentes en las trozas de calidad $A$ corresponden a rajaduras y a grietas por frío, y si se eliminaran las trozas que los presentan, el rendimiento aumenta a un 16\%, mientras en las trozas de calidad B existe una mayor presencia de heridas y pudriciones, defectos que reducen el rendimiento de las trozas ya que no permiten utilizar la madera que los presenta. Además, heridas de podas afectan el desarrollo de la madera, generándose grandes labios de madera de herida, afectando la madera que se desarrolla después de la herida (Shigo, 1994), por lo tanto, se debe tener especial cuidado sobre los factores que generan estos defectos en las plantaciones, para así aumentar los rendimientos y mejorar la calidad de la madera.

\section{Clasificación y Características de la Madera Aserrada}

En relación a la clasificación de la madera aserrada, esta evidenció algunos aspectos críticos porque las normas comúnmente adoptadas no resultan adecuadas para valorar la real posibilidad de uso de la madera obtenida del raleo, emergiendo la necesidad de realizar sistemas más adecuados o disponer de especificaciones técnicas ad hoc para la clasificación de este tipo de producto (Zanuttini et al., 2006), lo que es aún más notorio en el nogal común, donde la única clasificación existente, presentada por Berti et al. (2003) es apta únicamente para madera obtenida en rotaciones finales y no en raleos. Esto hace necesario el desarrollo de propuestas de clasificación para esta madera, que permitan facilitar y fomentar su comercialización tanto a nivel nacional como internacional.

Al analizar los volúmenes obtenidos por cada grado, se aprecia que el mayor porcentaje se presenta en el grado N2, con un 55,74 \% del volumen, seguido por el grado N3, con un 35,95\%. Si bien se esperaba que la mayor proporción del volumen se presentara en el grado con menor exigencia, esto no se verificó debido a que en este tipo de madera la presencia de defectos o el color hace variar mucho el precio en el mercado, entonces al dibujar las tablillas sobre las tapas se priorizó la calidad por sobre la cantidad de madera obtenida, por lo que un $65,05 \%$ de la madera aserrada obtenida pertenece a las mejores categorías, es decir, grados N1 y N2.

Respecto al tipo de defecto que afectó la clasificación de cada pieza según nivel (Figuras 6 y 7), se aprecia que en ambos niveles el principal defecto que limita la clasificación de las piezas es su dimensión, indicando que si bien existe la posibilidad de obtener piezas de mejores calidades, la limitante es el tamaño de las trozas, mientras el segundo defecto más recurrente correspondió a nudos con pudrición, lo que era esperable dado que en las plantaciones donde se obtuvieron las trozas, por ser experimentales, se realizaron distintas podas, algunas de las cuales facilitaron el ingreso de patógenos 0 problemas de cicatrización generados cuando fueron mal realizadas. Entre los defectos por aserrío, el más frecuente es la acanaladura, seguido por la arqueadura y finalmente la torcedura; la encorvadura casi no se presenta, por lo que el encastillado de la madera se debe realizar con el objetivo de evitar los primeros defectos mencionados.

Estéticamente las piezas aserradas obtenidas presentaron únicamente colores claros, entre el blanco y el grisáceo (Figura 8). No se observó duraminización en las trozas, lo que concuerda con lo indicado por Álamos (2003), quien encontró que la mayoría de las piezas obtenidas eran de colores claros y sin duraminización; Zanuttini et al. (2006) observaron en trozas de 7 años colores particularmente claros, pero con duraminización en algunos casos.

El tipo de colores obtenidos es altamente demandado en el mercado por su facilidad para teñir (JayAllemand et al., 1998), lo que sería una ventaja de las piezas obtenidas de raleo; al respecto, Zanuttini et al. (2006) indican que comercialmente son más apreciadas las chapas y madera aserrada de color claro (nogal rubio) respecto a madera semielaborada de tonalidad más intensa y variada (nogal colorido), pero se debe considerar que no solo la edad condiciona el color de la madera, ya que, como indicado por JayAllemand et al. (1998) y Zanuttini et al. (2006), el color de la madera depende también de la sustancia constitutiva de la pared celular y de la sustancia que compone el lumen celular, los cuales varían con la especie, la actividad fisiológica de la planta, las características del sitio (climas secos y terrenos arcillosos determinan coloraciones más oscuras), y a los procesos de transformación por envejecimiento del tejido celular, principalmente por duraminización. 
Considerando que Loewe-Muñoz et al. (2020) mostraron que en plantaciones mixtas de nogal común con cerezo común (Prunus avium L.) y olivo de Bohemia (Elaeagnus angustifolia L.) como especies acompañantes, se obtienen mayor altura (hasta $79 \%$ ) y DAP (hasta 53\%) que en plantaciones puras, así como daños fitosanitarios menos graves, se sugiere emplear este tipo de plantaciones para obtener trozas con mayor potencial de uso. La selección del sitio y de las especies acompañantes debe realizarse considerando tanto la calidad del sitio como la intensidad de manejo.

\section{CONCLUSIONES}

El estudio realizado evidenció que las piezas aserradas obtenidas poseen cierta utilización potencial, principalmente como decoración y embalaje, por lo que se recomienda realizar estudios de diseño de cajas y otros elementos para valorizar esta madera.

\section{REFERENCIAS}

Álamos, V. (2003). Evaluación Económica del aprovechamiento de madera de desecho de nogal común (Juglans regia L) como parquet. Tesis Ingeniero Forestal. Pontificia Universidad Católica de Chile, Escuela de Agronomía e Ingeniería Forestal. Santiago. $64 \mathrm{p}$.

Álvarez, D., Candano, F., Betancourt, Y., Rodriguez, J., Esteves, I., Díaz, A., Chavez, P. \& Fosado, O. (2005). Aprovechamiento Forestal en el Occidente de Cuba y su Impacto sobre el Medio Ambiente. Universidad de Pinar del Río. En: http://www.monografias.com/trabajos-pdf/aprovechamiento-forestal/ aprovechamiento-forestal.pdf. Consulta: 18 Julio, 2006.

Berti, S., Brunetti, M. \& Rescic L. (2003). Manuale sulla valutazione della quelità degli assortimenti legnosi ritraibili dalle specie legnose pregiate. Italia, C'è vita nel bosco.

Fassola, H., Fahler, J., Ferrere, P. \& Alegranza, D. (1999). Estudio de rendimiento en madera libre de nudos y variables que la determinan en Pinus taeda. Montecarlo, Francia. Consultado 18 Julio $2006 . \quad$ En: http://www.inta.gov.ar/montecarlo/info/documentos/forestales/25 Estudio rendimiento madera.pdf.

Góngora, E. \& Serrano, J. (2006). Pruebas de Rendimiento para Obtener Tablitas de Melina. (Kurú) Consultado 18 Julio 2006. Disponible en: http://www.itcr.ac.cr/revistakuru/pdf/Articulo\%201.pdf.

Jaime, N. (2006). Evaluación de aprovechamiento y propuesta de clasificación de madera de desecho de nogal común (Juglans regia L.). Tesis para optar al Título de Ingeniero Forestal, Universidad Santo Tomás.

Jay-Allemand, C., Janin, G. \& Charpentier. J.P. (1998). Characterization of Walnut woods for standardization. En: European Commission. Walnut: Productions of High Quality Timber in Europe. Up-to-date tools for tree adaptation, wood standards and management. W- Brains, Francia. Pp: 11-16.

Loewe, V. \& González, M. (2001). Nogal común: Una alternativa para producir madera de alto valor. Santiago, Chile, INFOR - FIA. $165 \mathrm{p}$.

Loewe-Muñoz, V., Balzarini, M y González, M. (2020). Pure and mixed plantations of Persian Walnut (Juglans regia L.) for high quality timber production in Chile, South America, Journal of Plant Ecology, 13. Pp: 12-19. https://doi.org/10.1093/ipe/rtz042.

Sevrin, E. \& Lemaire, J. (2004). Transformer le bois et vendre un produit fini. In Sevrin E, Lemaire J. Les guides du Sylviculteur: Le Châtaignier un arbre, un bois. 2a Ed. Francia. Instituto por le Développment Forestier. Pp: 317-326.

Shigo, A. (1994). Arboricultura Moderna Compendio. New Hampshire, Estados Unidos, Shigo and Trees, 152 p. Zanuttini, R., Cremonini, C., Brunetti, M. \& Berti, S. (2006). Caratterizzazione del tondame di noce e ciliegio. Sherwood, 120. Pp: 7-13. 